
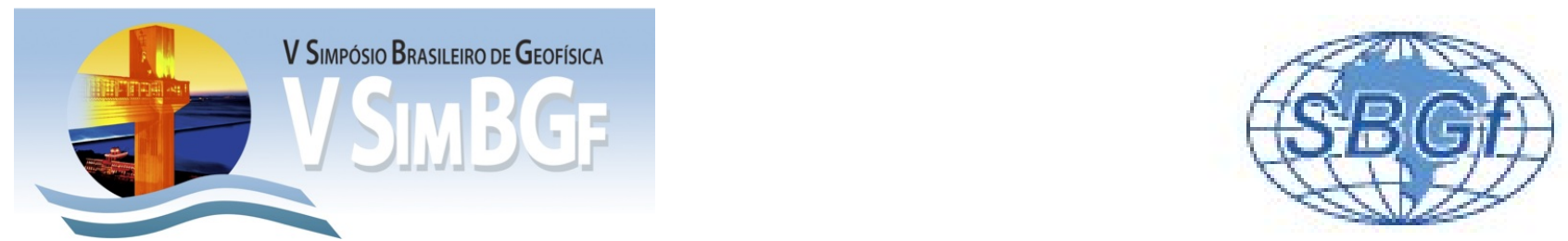

\title{
Análise de velocidade com migração residual de difrações pre- e pos-empilhamento no domínio da profudidade
}

\author{
Migration velocity analysis using residual diffraction moveout in the pre/post- \\ stack depth domain
}

Tiago A. Coimbra(IMECC-UNICAMP \& INCT-GP), J.J.S. de Figueiredo*(IG-UFPA \& INCT-GP), Jörg Schleicher (IMECC-UNICAMP \& INCT-GP) Amélia Novais (IMECC-UNICAMP \& INCT-GP) and Jessé Costa (IG-UFPA \& INCT-GP)

Copyright 2012, SBGf - Sociedade Brasileira de Geofísica Este texto foi preparado para a apresentação no V Simpósio Brasileiro de Geofísica, Salvador, 27 a 29 de novembro de 2012. Seu conteúdo foi revisado pelo Comitê Técnico do V SimBGf, mas não necessariamente representa a opinião da SBGf ou de seus associados. É proibida a reprodução total ou parcial deste material para propósitos comerciais sem prévia autorização da SBGf.

\section{RESUMO}

Eventos relacioanados a curvas de difrações contém informações diretas da velocidade do meio. Neste trabaIho, desevolvemos um método para melhoramento do modelo de velocidade e localização de pontos difratores baseado na análise de difrações não colapsadas no domínio da profundidade. O método usa um modelo de velocidade inicial arbitrário como parâmetro de entrada. Este método fornece a atualização do modelo de velocidade e localização de difratores como resultados principais. O algorítimo é baseado na focalização de raios de velocidades de remigração a partir de curvas de difrações migradas incorretamente. Estes raios de velocidades são construídos a partir de uma abordagem semelhante a de traçamento de raios aplicado a equação da onda imagem para continuação de velocidade. Depois da escolha dos eventos de difrações no domínio migrado os pontos difratores são localizados automaticamente. O método possui um custo computacional muito baixo. A viabilidade do nosso método é verificada a partir de um conjunto de exemplos númericos.

\section{ABSTRACT}

Diffraction events contain direct information on the medium velocity. In this work, we develop a method for migration velocity improvement and diffraction localization based on a moveout analysis of over or undermigrated diffraction events in the depth domain. The method uses an arbitrary initial velocity model as input. It provides an update to the velocity model and diffraction locations in the depth domain as a result. The algorithm is based on the focusing of remigration velocity rays from incorrectly migrated diffraction curves. These velocity rays are constructed from a ray-tracing like approach applied to the image-wave equation for velocity continuation. After picking the diffraction events in the migrated domain, the method has a very low computational cost, and the diffraction points are located automatically. We demonstrate the feasibility of our methods using a set of synthetic data examples.

\section{INTRODUCTION}

To correctly locate seismic reflectors in the subsurface, the migration velocity used in seismic processing needs to be as close as possible to the correct velocity of the reflector overburden. However, the possibilities to obtain the correct velocity is limited and not always achieved by conventional seismic processing (i.e., common-midpoint analysis). Therefore, the development of new techniques capable of improving the velocity model is still desirable.

In this work, we propose a method for velocity improvement and diffraction-point localization based on the localization and picking of residual moveout of incorrectly migrated diffraction events in the depth domain. Our analysis is performed over diffraction curves in depthmigrated common-offset (CO) profiles. At low computational cost, the method uses an approximate velocity model as an input and provides an update to the velocity. We propose three algorithms for the use of the residual diffraction moveout for velocity updating. All of them are based on depth remigration (Hubral et al., 1996a; Tygel et al., 1996; Hubral et al., 1996b; Schleicher et al., 1997, 2004) and give rise to automated methods for diffraction location that relies on the picking of over or undermigrated diffractions in the image. We demonstrate the feasibility of our method on numerical examples.

\section{THEORETICAL DESCRIPTION}

Let us consider a diffraction point at $\left(x_{d}, z_{d}\right)$ in a constantvelocity medium with velocity $v_{d}$. From the underlying arguments of depth remigration (Hubral et al., 1996b), 
it follows that the residual moveout of a diffraction event after depth migration with a wrong velocity $v_{0}$ is nothing else than the corresponding Huygens image-wave for depth remigration from velocity $v_{d}$ to $v_{0}$ (both $v_{0}$ and $v_{d}$ assumed to be constant). According to (Hubral et al., 1996b), the Huygens image-wave is the curve or surface of all points where a possible (reflection or diffraction) event at an image point $\left(x_{d}, z_{d}\right)$ might be placed when the migration velocity is changed from $v_{d}$ to $v_{0}$. Since a diffraction event migrated with the true velocity $v_{d}$ focuses at the true position $\left(x_{d}, z_{d}\right)$, its location after migration with a wrong velocity $v_{0}$ must be smeared over the Huygens image-wave. In the constant-offset depth domain, this is given by

$$
\frac{z^{2}}{v_{0}^{2}}+\frac{\left(x-x_{d}\right)^{2}}{v_{0}^{2}-v_{d}^{2}}=\frac{z_{d}^{2}}{v_{d}^{2}}+h^{2}\left(\frac{1}{v_{d}^{2}}-\frac{1}{v_{0}^{2}}\right)
$$

where $h$ is the half-offset.

Introducing the sign symbol $s=\operatorname{sgn}\left(v_{0}^{2}-v_{d}^{2}\right)=\operatorname{sgn}\left(v_{0}-\right.$ $v_{d}$ ) of the difference between the true and migration velocities, this equation can be rewritten as an ellipse or hyperbola,

$$
\frac{z^{2}}{b^{2}}+s \frac{\left(x-x_{d}\right)^{2}}{a^{2}}=1
$$

where the half-axes $a$ and $b$ are given by

$$
\begin{aligned}
a & =\frac{\sqrt{\left|v_{0}^{2}-v_{d}^{2}\right|} \sqrt{v_{0}^{2}\left(z_{d}^{2}+h^{2}\right)-v_{d}^{2} h^{2}}}{v_{d} v_{0}} \\
b & =\frac{\sqrt{v_{0}^{2}\left(z_{d}^{2}+h^{2}\right)-v_{d}^{2} h^{2}}}{v_{d}}
\end{aligned}
$$

As we can see from equation 2, when the migration velocity is smaller than the medium velocity, the undermigrated diffraction event follows an (approximate) hyperbola. On the other hand, when the migration velocity is higher than the medium velocity, the overmigrated diffraction event follows an (approximate) ellipse.

\section{Velocity updating}

Whenever the local velocity distribution at the diffraction point is reasonably well approximated by a constant average velocity, we can use the theoretical description in equation 2 to pick the incorrectly migrated diffraction events. We use the least-squares method to find the best-fitting hyperbola to describe an undermigrated diffraction event or the best-fitting ellipse for an overmigrated diffraction event. This provides estimates for the half-axes $a$ and $b$ as well as for horizontal coordinate of the apex, $x_{d}$.

From equations 3 we immediately recognize the first approach to find the velocity at diffraction point, $v_{d}$, as well as the depth location $z_{d}$. Solving equation system
3 for $v_{d}$ and $z_{d}$, we find

$v_{d}=\frac{v_{d} b}{\sqrt{b^{2}-s v_{d}^{2} a^{2}}} \quad$ and $\quad z_{d}=\sqrt{\frac{v_{d}^{2} h^{2}+b^{2}}{v_{0}^{2}}-h^{2}}$.

The horizontal coordinate of the diffractor is found from the fitting of the ellipse or hyperbola, which defines an estimate of $x_{d}$, according to equation 2 .

\section{Velocity updating using remigration trajectories}

There is a more sophisticated way of using the information contained in the residual diffraction moveout for a velocity updating procedure, which is less dependent on the assumption of constant velocity. It is based on the velocity continuation method, also known as imagewave equation for remigration. This is an imaginary continuation operation in which the seismic image is transformed continuously in the post-migration domain (Fomel, 1994, 2003; Hubral et al., 1996b; Sava e Fomel, 2003; Schleicher et al., 2004) as a function of velocity. Mathematically, it is described by a so-called imagewave equation. In the depth domain, this equation is given by

$$
\frac{\partial^{2} I}{\partial x^{2}}+\left(1+\frac{h^{2}}{z^{2}}\right) \frac{\partial^{2} I}{\partial z^{2}}-\frac{v}{z} \frac{\partial^{2} I}{\partial v \partial z}=0
$$

where $I=I(x, z, v)$ is a image-wave function representing the seismic image to be transformed, $x, z$ are the space variables and $v$ is the average velocity at an image point $(x, z)$. By applying a ray-theory-like approach to the remigration image-wave equation, ray-like trajectories can be found. These remigration trajectories are the set of positions where a selected reflection point can be found in a migrated image as a function of migration velocity.

\section{Remigration trajectory tracing}

In other words, taking into account only the kinematic part of depth remigration, we use an WKBJ-type approximation

$$
I(x, z, v)=A(x, z) F(v-V(x, z))
$$

to describe the seismic image. In equation 6 , amplitude factor $A$ represents the dynamic behavior, which we ignore, $F$ is the seismic wavelet and $V$ is image-wave eikonal describing the kinematic behavior. For depth remigration $V$ satisfies the image-eikonal equation,

$$
\left(\frac{\partial V}{\partial x}\right)^{2}+\left(1+\frac{h^{2}}{z^{2}}\right)\left(\frac{\partial V}{\partial z}\right)^{2}-\frac{V}{z} \frac{\partial V}{\partial z}=0 .
$$

For zero-offset $(h=0)$, the solution to equation 7 are circular arcs (Schleicher et al., 1997). For non-zerooffset, equation 7 can be solved using the method of 
characteristics (Courant e Hilbert, 1989) by transforming it into a hyper-surface $G$ as

$$
G(x, z, V, p, q)=p^{2}+\left(1+\frac{h^{2}}{z^{2}}\right) q^{2}-\frac{V}{z} q=0,
$$

where $p=\partial V / \partial x$ and $q=\partial V / \partial z$ are new variables. The method of characteristics consists of transforming the partial differential equation 7 into the following system of ordinary differential equations in terms of hypersurface $G$

$$
\begin{aligned}
\frac{d x}{d v} & =\lambda \frac{\partial G}{\partial p}=2 \lambda p \\
\frac{d z}{d v} & =\lambda \frac{\partial G}{\partial q}=\lambda\left(2\left(1+\frac{h^{2}}{z^{2}}\right) q-\frac{V}{z}\right) \\
\frac{d p}{d v} & =-\lambda\left(G_{x}+p G_{V}\right)=\lambda \frac{q p}{z} \\
\frac{d q}{d v} & =-\lambda\left(G_{z}+q G_{V}\right)=-\lambda\left(\frac{q}{z^{3}}\left(z V-q\left(2 h^{2}+z^{2}\right)\right)\right) \\
\frac{d V}{d v} & =-\lambda\left(p G_{p}+q G_{q}\right)=\lambda\left(p^{2}+\left(1+\frac{h^{2}}{z^{2}}\right) q^{2}\right)=1, \\
\lambda & =\left(p^{2}+\left(1+\frac{h^{2}}{z^{2}}\right) q^{2}\right)^{-1} .
\end{aligned}
$$

In the first five equations of system 9, $v$ could be any monotoneously increasing variable along the trajectory. For convenience, we have imposed the meaning of the independent variable to be the (average) velocity by setting the derivative of the image-eikonal $V$ with respect to $v$ to one. System 9 describes the remigration trajectories the image wave follows under variation of $v$. In other words, all variables involved in the propagation process are parameterized in terms of $v$, i.e., as $x=x(v), z=z(v), p=p(v), q=q(v)$, and $V=V(v)$. Since the second equation of system 9 depends explicitly on $v$, it is conceivable that this system can be used to trace remigration trajectories in inhomogeneous media, and for nonzero offset, where they are no longer circular arcs.

Initial conditions. To make use of system 9 for the tracing of the remigration trajectories, we need initial conditions for all of these variables. Let us assume that a remigration trajectory starts at an image-point $\left(x_{0}, z_{0}\right)$ obtained with velocity $v_{0}$. This defines the initial values for image-wave ray tracing as $x\left(v_{0}\right)=x_{0}, z\left(v_{0}\right)=z_{0}$ and $V\left(v_{0}\right)=v_{0}$. We still need the initial values $p\left(v_{0}\right)=$ $p_{0}$ and $q\left(v_{0}\right)=q_{0}$. These values satisfy the imageeikonal equation 8 at $v=v_{0}$, which can be recast into the form of an elliptic expression. The $p$ and $q$ values are obtained following

$$
p_{0}=-\frac{v_{0}}{z_{0}} \frac{D_{0}}{D_{0}^{2}+\alpha^{2}} \quad \text { and } \quad q_{0}=\frac{v_{0}}{z_{0}} \frac{1}{D_{0}^{2}+\alpha^{2}}
$$

where $\alpha^{2}=1+\frac{h^{2}}{z^{2}}$ and $D_{0}$ is the dip of the migrated event at the initial point $\left(x_{0}, z_{0}\right)$ of the remigration trajectory. These two equations complete the set of initial conditions needed for the tracing of the remigration trajectories using system 9 .

\section{RESULTS}

To investigate the influence of lateral inhomogeneity, we applied our method in a simple model depicted in Figure 1a. It contains ten diffraction points and a continuous reflector consisting of four straight elements with two different dips, buried in a constant-gradient velocity background with vertical and lateral variation, given by $v(x, z)=2000+0.4 x+0.6 z \mathrm{~m} / \mathrm{s}$. We generated zero-offset data by Kirchhoff modeling, simulating a zero-offset section with 200 source-receivers pairs spaced at $10 \mathrm{~m}$ covering an extension of $2000 \mathrm{~m}$. We used a Ricker wavelet of dominant frequency $25 \mathrm{~Hz}$. To these synthetic data we added random noise with a signal-to-noise ratio $(\mathrm{S} / \mathrm{N})$ of 10 with respect to the strongest diffraction event. These zero-offset data are depicted in Figure $1 \mathrm{~b}$ and the depth migrated image using a constant velocity of $1900 \mathrm{~m} / \mathrm{s}$ is shown in Figure 1c. The remigration trajectories (see the red lines
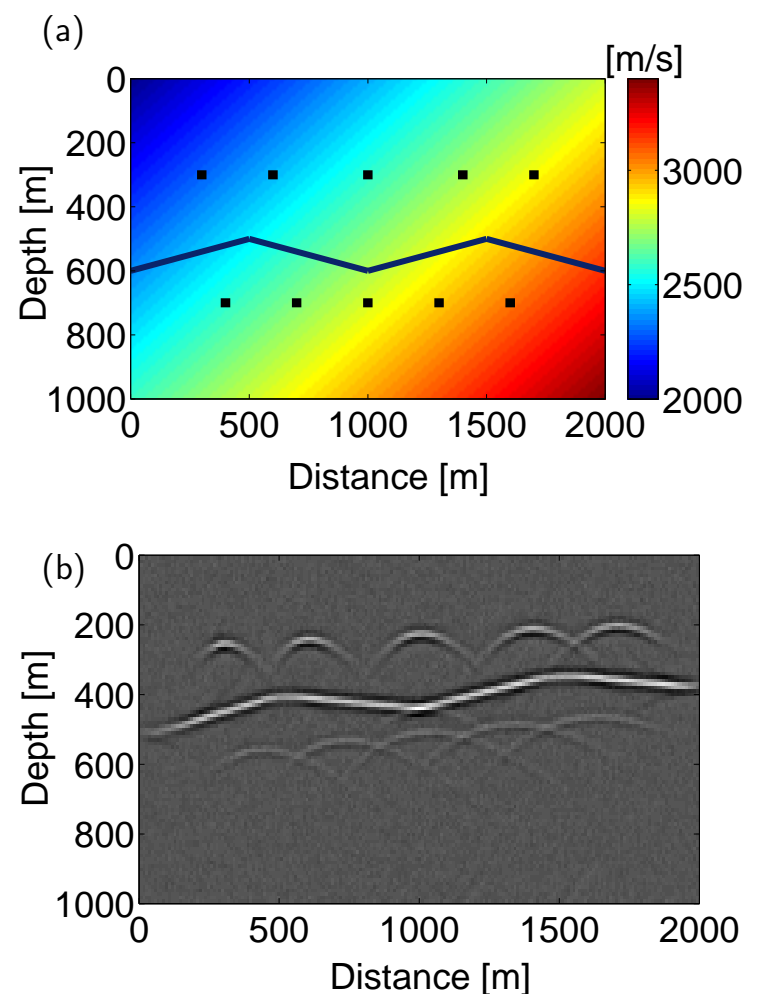

Figura 1: (a) Constant-gradient model, containing ten diffractors and a reflector.(b) Kirchoff depth migrated image using a constant velocity of $1900 \mathrm{~m} / \mathrm{s}$.

in Figure 2a) from the fitted diffraction events focus exactly at the true depths. In this example, even under a rather strong lateral and vertical velocity gradient, the method was capable of localizing all diffraction points 
with an error of $\pm 0.5 \%$ in the vertical direction and up to $\pm 1.0 \%$ in the horizontal direction. We used a linear least-squares fit to obtain an mean velocity model given by $v(x, z)_{\text {ave }}=2048+0.4 x+0.2 z \mathrm{~m} / \mathrm{s}$.

To invert this mean velocity model for interval velocity, we use that depth remigration averages the slowness (Schleicher et al., 2004). Figure $2 b$ shows the velocity model as constructed from inverting the average slowness, and Figure $2 c$ shows its relative error with respect to the true velocity model depicted in Figure 1c. As we can see, the error amounts to less than $4.5 \%$ at the borders of the model and is much lower at the center, where the actual velocity information is located.
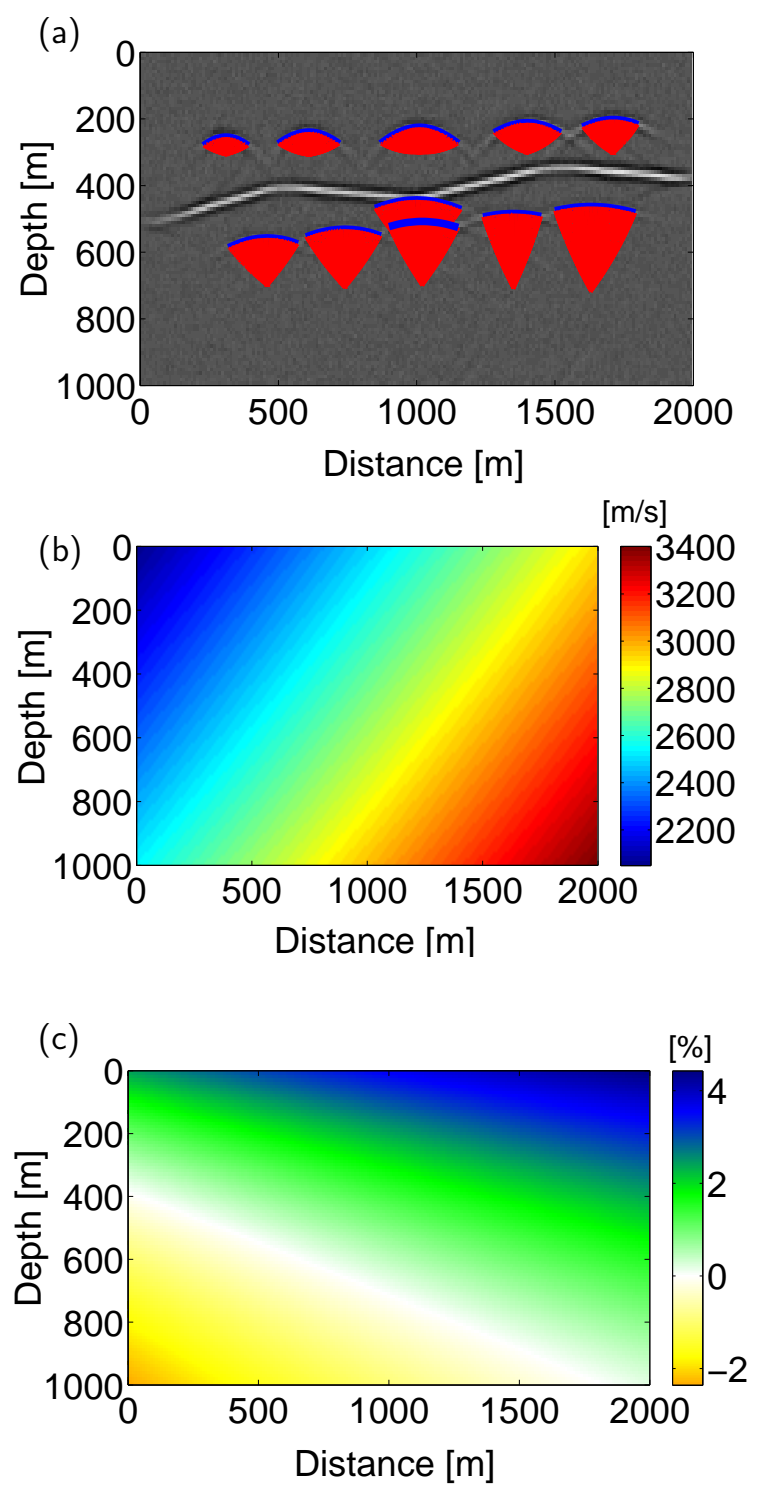

Figura 2: (a) Remigration trajectories collapsing in the depth point where depth migration should locate the diffractor if the correct velocity model had been used. (b) Interval velocity model from average slowness. (c) Relativevelocitatyserror example, we use the reduced Sigs-
bee2B model (see Figure 3a). This model has a velocity background of $v(z)=1520+0.3\left(z-z_{\text {seafloor }}\right)(\mathrm{m} / \mathrm{s})$ for each layer, where the argument $z$ is the depth below the water bottom. The corresponding synthetic seismograms were obtained using acoustic finite-difference modeling (see Figure $3 \mathrm{~b}$ ). The zero-offset section displayed in Figure 3 consists of 400 traces separated by $45.75 \mathrm{~m}$. The total size of each trace is $8 \mathrm{~s}$ and the number of sample per trace is 1001 , resulting in a time sample rating of $0.008 \mathrm{~s}$. Figure $3 \mathrm{c}$ shows the depth migrated section obtained with SU's Gaussian beam migration with a constant velocity of $1500 \mathrm{~m} / \mathrm{s}$. This figure also shows the remigration trajectories (in red) in emanating out from uncollapsed diffraction curves (in blue). In this first iteration the points where the remigration trajectories are crossing represent a first guess for the diffraction locations.

In Figure 4a, we display the Gaussian-beam depth-migrated section after the second iteration with remigration trajectories in red. Here we can note that the number of diffractions that where required to furter update the velocity model is reduced to three diffraction points. After the third update of the velocity model obtained from the remigration operation, using the diffractions indicated in the depth migrated section of Figure $4 a$, we reach the velocity model of the third iteration, which is shown in Figure 4b. The resulting Gaussian-beam depth migrated section can be seen in Figure 4c.

It is important to note that a very small number of identified and picked diffractions in only three iterations was sufficient to estimate a very good velocity model of the region above the salt body. Using only five diffraction curves in the first iteration, and three diffractions in the two subsequent iterations, we were capable of producing a high-quality image of the sedimentary region. As shown in Figure 4c, the top of salt and the prominent sediment reflectors were imaged with a positioning error of less than $1 \%$. It must be stressed that even in a model as large as the Segsbee2b model, our method was effectively fast and of a very low computational cost.

\section{CONCLUSIONS}

We have developed a new method for diffraction imaging and velocity model improvement in the depth domain. The method uses the moveout of unfocused diffraction events in a migrated seismic section. The focusing of remigration trajectories originating from these events is used to determine the correct location of the diffractor and the associated velocity value. The method is computationally undemanding.

In our numerical examples, the method worked satisfactorily. In a first constant-gradient example, all diffraction points were located with an error of less than 
(a)

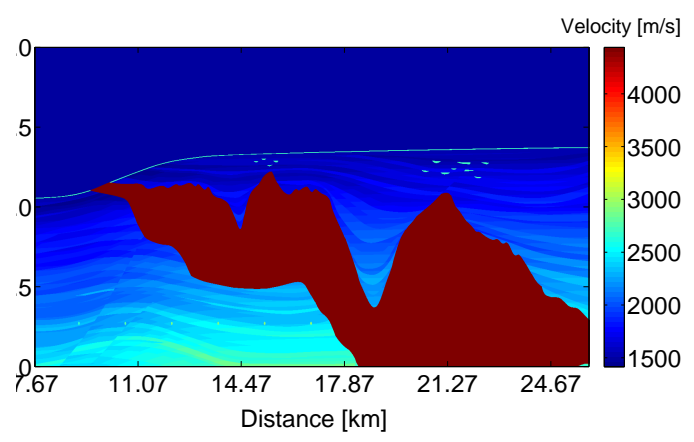

(b)

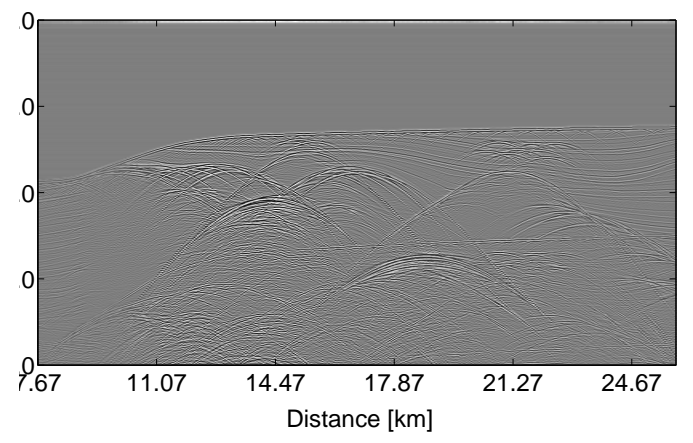

(c)

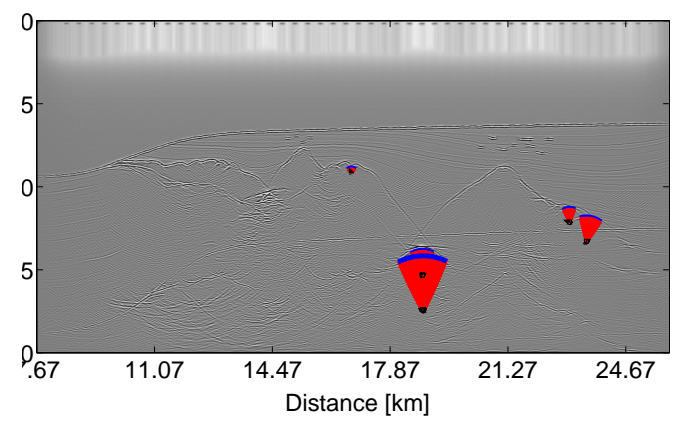

Figura 3: (a) Reduced stratigraphic Sigsbee2b velocity model. (b) Zero-offset time section. (c) Migrated section using constant velocity of $1500 \mathrm{~m} / \mathrm{s}$ using gaussian beam depth migration. The red curves are the remigration trajectories.

$1 \%$. The extracted velocity models were acceptably accurate, with an error of less than $4.5 \%$. Combining results, we could find a reasonable estimate of the lateral velocity variation in the vicinity of the diffraction point. In a second, more realistic numerical example using the Segsbee2b data set, three iterations using at most five diffraction points, the method produced a velocity model that was capable of correctly imaging the sedimentary region between the sea bottom and the top of salt reflectors.

\section{ACKACKNOWLEDGEMENTS}

The authors thank SMAART-JV for providing the Segs- (a)

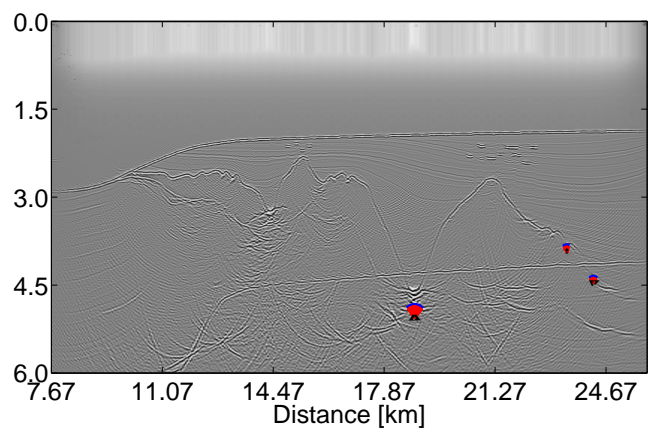

(b)

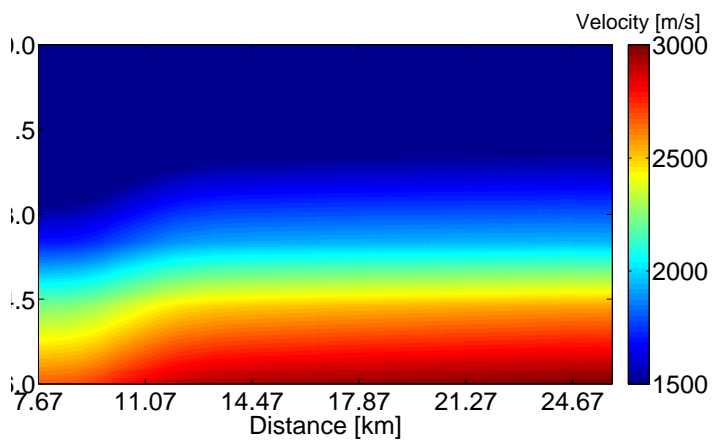

(c)

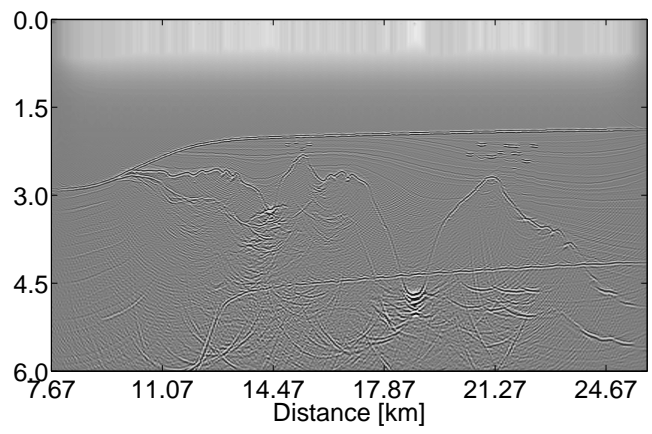

Figura 4: (a) Migrated section from second iteration (and with remigration trajectories). (b) Velocity model from third iteration.(c) Migrated section using velocity model from third direction.

bee2b data. This work was kindly supported by the Brazilian agencies CAPES, FINEP, and CNPq, as well as Petrobras and the sponsors of the Wave Inversion Technology (WIT) Consortium.

\section{REFERÊNCIAS}

Courant, R., e Hilbert, D., 1989, Methods of mathematical physics:, volume II Wiley-Interscience.

Fomel, S., 1994, Method of velocity continuation in the problem of seismic time migration: Russian Geology and Geophysics, 35, no. 5, 100-111. 
Fomel, S., 2003, Velocity continuation and the anatomy of residual prestack time migration: Geophysics, 68, no. $5,1650-1661$.

Hubral, P., Schleicher, J., e Tygel, M., 1996a, A unified approach to 3-d seismic reflection imaging - part i: Basic concepts: Geophysics, 61, no. 3, 742-758.

Hubral, P., Tygel, M., e Schleicher, J., 1996b, Seismic image waves: Geoph. J. Int., 125, 431-442.

Sava, P. C., e Fomel, S. B., 2003, Angle-domain common image gathers by wavefield continuation methods: Geophysics, 68, no. 5, 1650-1661.

Schleicher, J., Hubral, P., Tygel, M., e Jaya, M. S., 1997, Minimum apertures and Fresnel zones in migration and demigration: Geophysics, 62, no. 2, 183194.

Schleicher, J., Novais, A., e Munerato, F. P., 2004, Migration velocity analysis by depth image-wave remigration: First results: Geophysical Prospecting, 52, 559-573.

Tygel, M., Schleicher, J., e Hubral, P., 1996, A unified approach to 3-d seismic reflection imaging - part ii: Theory: Geophysics, 61, no. 3, 759-775. 